\title{
Correction to: Three new ent-abietane diterpenoids from the roots of Euphorbia fischeriana and their cytotoxicity in human tumor cell lines
}

\author{
Minghui $\mathrm{Li}^{1} \cdot$ Fang He$^{1} \cdot$ Yuan Zhou ${ }^{1} \cdot$ Meigui Wang $^{1} \cdot$ Pingde Tao $^{1} \cdot$ Qingmei Tu$^{1} \cdot$ Guanghui Lv $^{1} \cdot$ \\ Xintao Chen ${ }^{1,2}$
}

Published online: 5 September 2020

(c) The Pharmaceutical Society of Korea 2020

\section{Correction to: The Pharmaceutical Society of Korea https://doi.org/10.1007/s12272-019-01151-y}

The article "Three new ent-abietane diterpenoids from the roots of Euphorbia fischeriana and their cytotoxicity in human tumor cell lines", written by Minghui Li, Fang He, Yuan Zhou, Meigui Wang, Pingde Tao, Qingmei Tu, Guanghui Lv, Xintao Chen, was originally published electronically on the publisher's internet portal (currently SpringerLink) on 17 April 2019 with open access. With the author(s)' decision to step back from Open Choice, the copyright of the article changed on 4 September 2020 to (C) The Pharmaceutical Society of Korea 2020 and the article is forthwith distributed under the terms of copyright. The original article has been corrected.

Publisher's Note Springer Nature remains neutral with regard to jurisdictional claims in published maps and institutional affiliations.

The original article can be found online at https://doi. org/10.1007/s12272-020-01267-6.

Guanghui Lv

lvguanghui0725@163.com

Xintao Chen

m18772836076@163.com

1 Department of Pharmacy, Taihe Hospital, Hubei University of Medicine, Shiyan 442000, People's Republic of China

2 Hubei Key Laboratory of Natural Medicinal Chemistry and Resource Evaluation, School of Pharmacy, Tongji Medical College, Huazhong University of Science and Technology, Wuhan 430030, People's Republic of China 